\title{
Antioxidant and anti-inflammatory effects of intravenously injected adipose derived mesenchymal stem cells in dogs with acute spinal cord injury
}

Yongsun Kim, Sung-ho Jo, Wan Hee Kim and Oh-Kyeong Kweon ${ }^{*}$

\begin{abstract}
Introduction: Mesenchymal stem cells can potentially be used in therapy for spinal cord injury (SCl). Methylprednisolone sodium succinate (MPSS) has been used as a scavenging agent in acute SCI treatment, but its use no longer recommended. This study aimed to identify ways to reduce the usage and risk of high doses of glucocorticoid steroids, and determine whether AD-MSCs could be used as an early alternative treatment modality for acute $\mathrm{SCl}$.

Methods: Sixteen adult beagle dogs with SCI were assigned to four treatment groups: control, MPSS, AD-MSCS, and AD-MSCS + MPSS. Additionally, one dog was used to evaluate the distribution of AD-MSCs in the body after injection. AD-MSCs $\left(1 \times 10^{7}\right.$ cells) were injected intravenously once a day for 3 days beginning at 6 hours post-SCI. MPSS was also injected intravenously according to the standard protocol for acute SCl. A revised Tarlov scale was used to evaluate hindlimb functional recovery. The levels of markers for oxidative metabolism (3-nitrotyrosine, 4-hydroxynonenal, and protein carbonyl) and inflammation (cyclooxygenase-2, interleukin-6, and tumor necrosis factor-a) were also measured.

Results: At 7 days post-treatment, hindlimb movement had improved in the AD-MSCs and AD-MSCs + MPSS groups; however, subjects in the groups treated with MPSS exhibited gastrointestinal hemorrhages. Hematoxylin and eosin staining revealed fewer hemorrhages and lesser microglial infiltration in the AD-MSCs group. The green fluorescent protein-expressing AD-MSCs were clearly detected in the lung, spleen, and injured spinal cord; however, these cells were not detected in the liver and un-injured spinal cord. Levels of 3-nitrotyrosine were decreased in the MPSS and AD-MSCs + MPSS groups; 4-hydroxynenonal and cyclooxygenase-2 levels were decreased in all treatment groups; and interleukin-6, tumor necrosis factor-a, and phosphorylated-signal transducer and activator transcription 3 levels were decreased in the AD-MSCs and AD-MSCs + MPSS groups.
\end{abstract}

Conclusion: Our results suggest that early intravenous injection of AD-MSCs after acute SCI may prevent further damage through enhancement of antioxidative and anti-inflammatory mechanisms, without inducing adverse effects. Additionally, this treatment could also be used as an alternative intravenous treatment modality for acute SCl.

Keywords: Antioxidant, Anti-inflammatory, Spinal cord injury, Mesenchymal stem cells, Dog

\footnotetext{
* Correspondence: ohkweon@snu.ac.kr

BK21 PLUS Program for Creative Veterinary Science Research, Research

Institute for Veterinary Science and College of Veterinary Medicine, Seoul

National University, 1 Gwanak-ro, Gwanak-gu, Seoul 151-742, South Korea
} 


\section{Introduction}

Spinal cord injury (SCI) results from the primary mechanical damage that occurs when structural thresholds are surpassed, which in turn leads to immediate physical and biochemical alterations. These alterations are followed by a secondary injury mechanism that includes a variety of vascular, biochemical, and cellular processes [1]. SCI is generally followed by immediate hematoma formation and oxidative and inflammatory responses. These events involve enzyme activation, mediator release, inflammatory cell migration, glial activation, and neuronal tissue degradation $[2,3]$. Hemorrhages cause the extracellular spaces in the spinal cord to become exposed to hemoglobin and its breakdown products, which subsequently results in free radical generation. Infiltrating neutrophils can directly damage tissue by producing reactive oxygen species and secreting proinflammatory mediators [4]. Moreover, microglial activation is involved in clearing the hematoma, and activated microglia secrete a variety of cytokines. Following SCI, the progression of these events inhibits axonal regeneration in the central nervous system, such that patients are unable to recover from the neurological damage.

The neuroprotective agent methylprednisolone sodium succinate (MPSS) has been widely used in the clinical treatment of acute SCI $[5,6]$. However, recent retrospective cohort studies have demonstrated the lack of a statistically significant difference between clinical outcomes in SCI patients treated with and without MPSS [7]. The therapeutic effect of MPSS on SCI therefore remains controversial. As the current standard effective therapeutic agent for the clinical treatment of acute SCI, MPSS has been shown to alleviate secondary injury by decreasing inflammation and spinal cord ischemia, as well as by inhibiting cell membrane lipid peroxidation (LP). However, administration of high doses of glucocorticoid steroids causes many complications, including increased incidence of infection, pneumonia, pressure sores, gastrointestinal bleeding, and deep vein thrombosis [8].

Much of the current research on SCI is focused on developing treatment methods that limit or prevent secondary injury. Minimizing secondary injury is generally achieved by ensuring adequate perfusion and oxygenation and by ancillary administration of neuroprotective agents for improving clinical signs. Transplantation of mesenchymal stem cells (MSCs) has been shown to provide neuroprotection, increase neuronal regeneration, and ameliorate the clinical signs of SCI $[9,10]$. In these studies, the transplanted MSCs not only improved the inflammatory environment and enhanced the survival of endogenous nerve cells, but also reduced fibrosis formation and were able to partially differentiate into neural cells $[9,11]$.

We hypothesized that adipose-derived MSCs (ADMSCs) intravenously transplanted into animals with acute
SCI would alleviate inflammation at the injured site because of their antioxidant and anti-inflammatory properties. Based on our hypothesis, administration of AD-MSCs should ultimately result in less scar tissue formation, aid ingrowth of neural progenitor cells, and improve limb function. The present study was conducted to reduce the usage and risk of high doses of glucocorticoid steroids, and to determine whether AD-MSCs could be used as an early alternative treatment modality for acute SCI.

\section{Methods}

\section{Isolation and culture of canine AD-MSCs}

MSCs derived from the adipose tissue of canine hip fat were isolated and characterized [11]. Specifically, adipose tissue was collected aseptically from the subcutaneous fat of a 2-year-old beagle dog under anesthesia. Tissues were washed with phosphate-buffered saline (PBS), minced, and digested with collagenase type I $(1 \mathrm{mg} / \mathrm{ml}$; Sigma-Aldrich, St. Louis, MO, USA) at $37{ }^{\circ} \mathrm{C}$ for $30-60$ minutes with intermittent shaking. The suspension was filtered through a $100 \mu \mathrm{m}$ nylon mesh and centrifuged to separate floating adipocytes from stromal cells. Preadipocytes in the stromal vascular fraction were plated at 8000-10,000 cells $/ \mathrm{cm}^{2}$ in T175 culture flasks containing Dulbecco's modified Eagle's medium (DMEM; Gibco-BRL, Grand Island, NY, USA) supplemented with $3.7 \mathrm{~g} / \mathrm{l}$ sodium bicarbonate, $1 \%$ penicillin and streptomycin, $1.7 \mathrm{mM}$ L-glutamine, $0.1 \mathrm{mM} \beta$-mercaptoethanol, and $10 \%$ fetal bovine serum (FBS). The cells were incubated in a humidified atmosphere at $37{ }^{\circ} \mathrm{C}$ with $5 \% \mathrm{CO}_{2}$. Unattached cells and residual nonadherent red blood cells were removed after 24 hours using a PBS wash, and the cell medium was replaced every 2 days. At passage 3, the cells were used for the following experiments.

\section{Animals}

Seventeen healthy 2-3-year-old beagle dogs weighing $8.5 \pm 2.2 \mathrm{~kg}$ were used in the study. All dogs were clinically judged to be in good health and to have a normal neurological status. During the experiment, all dogs were cared for in accordance with the animal care guidelines of the Institute of Laboratory Animal Resources at Seoul National University, Korea. Sixteen dogs were assigned to four groups based on the treatments: control (no treatment after SCI; $n=4$ ), MPSS (administration of MPSS after SCI; $n=4$ ), AD-MSCs (administration of AD-MSCs; $n=4$ ), and AD-MSCs + MPSS (administration of both AD-MSCs and MPSS; $n=4$ ). One additional dog was used to evaluate distribution of MSCs in the body after injection. The Institutional Animal Care and Use Committee of Seoul National University approved the experimental design (SNU-111102-8). 


\section{Induction of SCI}

SCI was induced using a previously described balloon compression method [10]. Briefly, the dogs were medicated and anesthetized with tramadol $(4 \mathrm{mg} / \mathrm{kg}$ intravenously, Toranzin; Samsung Pharm. Ind. Co., Seoul, Korea), propofol $(6 \mathrm{mg} / \mathrm{kg}$ intravenously, Provive $1 \%$; Claris Lifesciences, Ahmedabad, India), and atropine sulfate (0.05 mg/kg subcutaneously, Atropine; Jeil Pharm., Yongin, Korea). Anesthesia was maintained with isoflurane (Forane solution; Choongwae Pharm. Co., Seoul, Korea) at a minimum alveolar concentration (MAC) of 1.5 throughout the procedure. Electrocardiography, pulse oximetry, respiratory gas analysis, and rectal temperature measurement were performed using an anesthetic monitoring system (Datex-Ohmeda S/5; GE Healthcare, Little Chalfont, UK). The dogs were suspended in a ventral recumbent position, and hemilaminectomy was performed through a left paramedian approach at the fourth lumbar segment (L4). A hole of 3-5 $\mathrm{mm}$ was made in the left vertebral arch at L4 using a high-speed pneumatic burr, and a 4French embolectomy catheter (Edwards Lifesciences, Irvine, CA, USA) was inserted into the hole. Under fluoroscopic guidance, the balloon catheter was advanced until the tip was positioned at the cranial margin of the first lumbar segment (L1) vertebral body. The balloon was then inflated by injecting $50 \mu \mathrm{l} / \mathrm{kg}$ contrast agent (Omnipaque; GE Healthcare) diluted with saline in a 50:50 proportion. The balloon's positioning was confirmed using fluoroscopy. According to a previous study [9], this SCI model occludes more than $85 \%$ of the spinal canal, as confirmed by computed tomography. Following induction of the injury, the soft tissue and skin were closed using standard methods. The balloon was fixed with a Chinese finger-type suture and removed after 6 hours. After the operation, the dogs were monitored in an ICU, and manual bladder expression was performed at least three times daily if needed.

\section{Administration of MPSS and AD-MSCs}

Administration of MPSS and AD-MSCs was initiated following removal of the embolectomy catheter. MPSS (30 mg/kg, Methysol; Kunhwa Pharmaceutical Co., Seoul, Korea) was bolus injected into the cephalic vein and followed by continuous rate infusion $(5.4 \mathrm{mg} / \mathrm{kg} /$ hour) for the next 47 hours [6]. A suspension of $1 \times 10^{7}$ allogenic AD-MSCs in $10 \mathrm{ml}$ lactated Ringer's solution was administered intravenously once a day for 3 successive days.

\section{Labeling and tracking of AD-MSCs}

Twenty-four hours before transfection, $4 \times 10^{6}$ HEK293 cells were seeded into a $100 \mathrm{~mm}$ dish. The following day, a lentiviral packaging mix (System Biosciences, San Diego, CA, USA) encoding viral proteins Gag-Pol, Rev, and VSV$G$ and lentiviral transgene plasmids were transfected into each well for lentivirus production using Turbofect (Thermo Scientific, Waltham, MA, USA). Green fluorescent protein (GFP)-expressing virus particles were collected and transduced into AD-MSCs at passage 1. After the AD-MSCs reached $90 \%$ confluence, the cells were selected by puromycin ( $3 \mu \mathrm{g} / \mathrm{ml}$; Gibco-BRL). Approximately $30 \%$ of cells were successfully transduced after puromycin selection. The AD-MSCs were subcultured, and passage 3 cells were used for the following experiments. The GFP-labeled AD-MSCs were intravenously injected after SCI as already described. The dog was sacrificed at 7 days after transplantation and tissue samples from the lung, liver, spleen, normal spinal cord, and injured spinal cord were collected. GFP protein was identified in each specimen using western blot analysis. The injured spinal cord was fixed in a $10 \%$ formalin solution and embedded in paraffin. Longitudinal sections were made and the tissue was stained with 4',6-diamidino-2phenylindole (DAPI, 1:100; Sigma-Aldrich) to identify nuclei. Slides were observed using a fluorescent microscope.

\section{Clinical assessment}

Behavioral assessments were performed on days 2, 4, and 7 after the operation to evaluate functional recovery of the hind limbs. Each dog was videotaped from both sides and behind during the neurologic examination. Using video footage and the revised Tarlov scale [12], the dogs' gaits were scored independently by two individuals blinded to the experimental condition. The animal's vital signs and operation site were also checked. A fecal occult blood test (Cell Biolabs, Inc., San Diego, CA, USA) was performed to evaluate any adverse gastrointestinal effects of intravenous administration of $\mathrm{AD}$ MSCs and/or MPSS.

\section{Histopathological assessment}

The dogs were sacrificed through intravenous injection of potassium chloride under general anesthesia at 7 days after transplantation. The spinal cord from the 12th thoracic segment (T12) to the third lumbar segment (L3) was extracted by dissection. Each section was then placed in $10 \%$ sucrose/PBS at $4{ }^{\circ} \mathrm{C}$ for 12 hours and subsequently immersed in $20 \%$ sucrose solution overnight at $4{ }^{\circ} \mathrm{C}$. The sample was divided into two parts longitudinally. One-half of each section was immediately frozen with liquid nitrogen for western blot analysis and enzyme-linked immunosorbent assay (ELISA) tests. The other half was embedded in optimal cutting temperature compound (Surgipath; Leica Biosystems Richmond, Inc., Richmond, IL, USA), frozen, and cut longitudinally into $12 \mu \mathrm{m}$ sections with a cryomicrotome. These sections were mounted on silane-coated glass slides and stained with hematoxylin and eosin (H\&E) or Luxol fast blue stain. The hemorrhagic area was measured by tracing the 
hemorrhagic margin and calculated using an image analysis program (Image); NIH, Bethesda, MD, USA). The number of microglia was counted manually in the high-powered field from five randomly selected areas of the injured area margin.

\section{Oxidant metabolite assessment}

The peroxynitrite (PN) formation of 3-nitrotyrosine (3NT), the LP product 4-hydroxynenonal (4-HNE), and the protein oxidation-derived protein carbonyls (PC) were used as markers for oxidative damage. The level of each oxidation product (3-NT, 4-HNE, and PC) was assessed using ELISA kits (Cell Biolabs, Inc.). The samples were placed into the wells of the 3-NT, 4-HNE, and $\mathrm{PC}$ conjugate-coated plates and incubated at room temperature for 10 minutes. After incubation, the secondary antibody was added to each of the wells, incubated at room temperature for 1 hour, washed three times with washing buffer, and incubated with secondary antibodyhorseradish peroxidase conjugate for 1 hour. The wells were washed, the substrate solution added for color change, and absorbance measured at $450 \mathrm{~nm}$.

\section{Western blot analysis}

The organ tissues and the frozen half of each injured spinal cord specimen were used for western blot analysis. Briefly, the tissue was washed twice with PBS, and then homogenized with a sonicator in lysis buffer $(20 \mathrm{mM}$ Tris at $\mathrm{pH}$ 7.5, $1 \mathrm{mM}$ ethylenediaminetetraacetic acid, $1 \mathrm{mM}$ ethylene glycol tetraacetic acid, $1 \%$ Triton X-100, $1 \mathrm{mg} /$ $\mathrm{ml}$ aprotinin, $1 \mathrm{mM}$ phenylmethylsulfonylfluoride, $0.5 \mathrm{mM}$ sodium orthovanadate) on ice for 30 minutes. Lysates were cleared by centrifugation (10 minutes at $15,000 \mathrm{rpm}, 4^{\circ} \mathrm{C}$ ), and protein concentrations were determined using the Bradford method [13]. Equal amounts of protein $(20 \mu \mathrm{g})$ were resolved by electrophoresis on $10 \%$ sodium dodecyl sulfate-polyacrylamide gels and transferred to polyvinylidene fluoride membranes. Membrane blots were washed with TBST (10 mM Tris-HCl, pH 7.6, $150 \mathrm{mM} \mathrm{NaCl}, 0.05 \%$ Tween-20), blocked with $5 \%$ skimmed milk for 1 hour, and incubated with the appropriate primary antibodies at the recommended dilutions. The antibodies used included antibodies against actin (A3853; Sigma-Aldrich), GFP (MA515256; Thermo Scientific), interleukin (IL)-6 (ab6672; Abcam, Cambridge, UK), and cyclooxygenase-2 (COX-2, sc-7951), tumor necrosis factor alpha (TNF $\alpha, \mathrm{sc}-1350)$, phosphorylated signal transducer and activator of transcription 3 (pSTAT3, sc-8001R), $\beta 3$-tubulin (sc-69966), glial fibrillary acidic protein (GFAP, sc-65343), and galactosylceramidase (GalC, sc67352) (Santa Cruz Biotechnology, Santa Cruz, CA, USA). The primary antibodies (1:1000) were diluted in TBST. The membrane was then washed, and the primary antibodies were detected with goat anti-rabbit IgG or goat anti-mouse IgG conjugated to horseradish peroxidase
(1:5000; Invitrogen, Waltham, MA, USA). Bands were visualized using enhanced chemiluminescence (Invitrogen).

\section{Statistical analysis}

All results are expressed as mean \pm standard deviation (SD). Statistical analysis was performed using a commercially available statistical software program (SPSS Statistics, version 21.0; IBM Corp., Armonk, NY, USA). In all experiments, Kruskal-Wallis tests were followed by Mann-Whitney $U$ tests to compare between groups. $P<0.05$ was considered significant.

\section{Results}

\section{Clinical assessment}

All experimental dogs had no movement and muscle tone in the hind limbs after SCI. However, subjects in the ADMSCs group had significantly enhanced motor function compared with those in the control group at 7 days post treatment $(P<0.05$; Fig. 1 , Table 1$)$. In addition, gastrointestinal hemorrhage was not observed in the control and the AD-MSCs groups but was observed in the MPSS and AD-MSCs + MPSS groups until 4 days post SCI (Table 2). Additionally, one in four dogs in the AD-MSCs + MPSS group and one in three dogs in the MPSS group exhibited gastrointestinal hemorrhages at 4 days post SCI. Other adverse effects such as wound infections or delayed healing were not observed in any of the treatment groups.

\section{Distribution of AD-MSCs}

To determine the engraftment of intravenously injected AD-MSCs, GFP was examined at 7 days after administration of GFP-expressing AD-MSCs. GFP was detected in the lung, spleen, and injured spinal cord, but was not detected in the liver and uninjured spinal cord (Fig. 2a). The expression of GFP in the lung was relatively higher

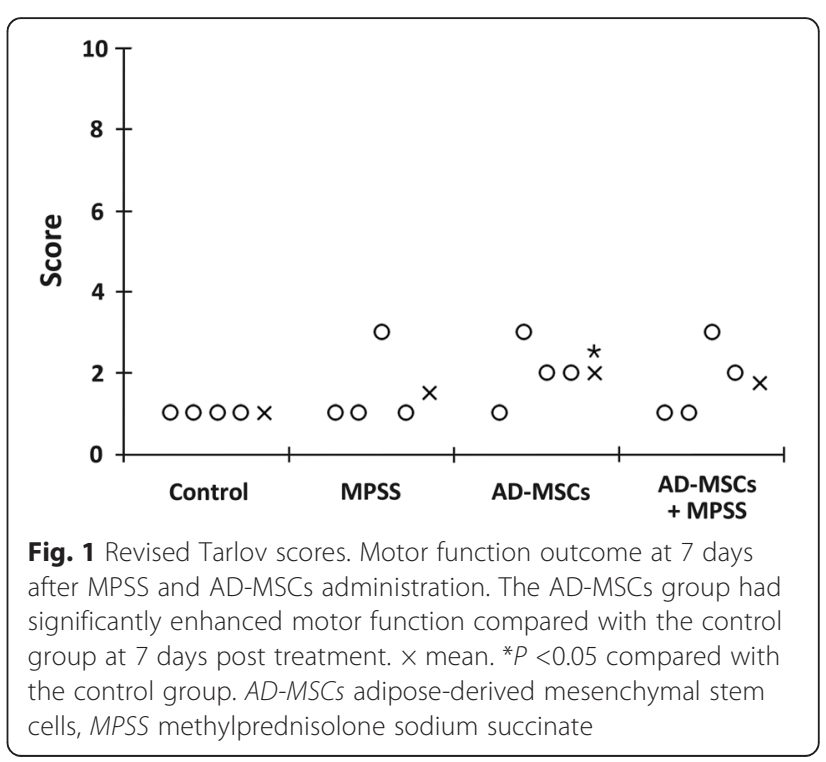


Table 1 Revised Tarlov scores

\begin{tabular}{lllll}
\hline Group & $n$ & Mean & Standard deviation & $P$ value \\
\hline Control & 4 & 1.00 & 0 & 0.317 \\
MPSS & 4 & 1.50 & 1.00 & \\
Control & 4 & 1.00 & 0 & 0.046 \\
AD-MSCs & 4 & 2.00 & 0.82 & \\
Control & 4 & 1.00 & 0 & 0.131 \\
AD-MSCs + MPSS & 4 & 1.75 & 0.96 & \\
MPSS & 4 & 1.50 & 1.00 & 0.350 \\
AD-MSCs & 4 & 2.00 & 0.82 & 0.617 \\
MPSS & 4 & 1.50 & 1.00 & \\
AD-MSCs + MPSS & 4 & 1.75 & 0.96 & 0.647 \\
AD-MSCS & 4 & 2.00 & 0.82 & \\
AD-MSCs + MPSS & 4 & 1.75 & 0.96 &
\end{tabular}

$P$ values were calculated using the Mann-Whitney $\mathrm{U}$ test

AD-MSCs adipose-derived mesenchymal stem cells, MPSS methylprednisolone sodium succinate

than other organ tissues. GFP-labeled AD-MSCs were observed in the epicenter of the injured spinal cord (Fig. 2b).

\section{Histopathological assessment}

Seven days after the initial transplantation, the average damaged lesion size per section of groups was $68.4 \pm$ $4.5 \mathrm{~mm}^{2}$. There were no significant differences in the area of the lesion among the four groups (Fig. 3). In the low-powered field, H\&E-stained sections from all groups showed severe hemorrhage and infiltration of microglial cells in the injured part of the spinal cord. In the high-powered field, an injured spinal cord parenchyma composed of demyelinated neurons, cell debris, and mild fibrosis as well as hemorrhage was observed. Luxol fast blue staining also showed demyelination in the injured area. However, hemorrhages were less commonly observed in the AD-MSCs group and a lower inflammatory response was observed in the AD-MSCs and AD-MSCs + MPSS groups (Fig. 4).

Table 2 Percentage of gastrointestinal hemorrhage

\begin{tabular}{llll}
\hline Group & \multicolumn{3}{l}{ Time after treatment } \\
\cline { 2 - 4 } & 2 days & 4 days & 7 days \\
\hline Control & $0 \%(0 / 4)$ & $0 \%(0 / 4)$ & $0 \%(0 / 4)$ \\
MPSS & $100 \%(4 / 4)$ & $75 \%(3 / 4)$ & $0 \%(0 / 4)$ \\
AD-MSCs & $0 \%(0 / 4)$ & $0 \%(0 / 4)$ & $0 \%(0 / 4)$ \\
AD-MSCS + MPSS & $100 \%(4 / 4)$ & $25 \%(1 / 4)$ & $0 \%(0 / 4)$
\end{tabular}

AD-MSCs adipose-derived mesenchymal stem cells, MPSS methylprednisolone sodium succinate
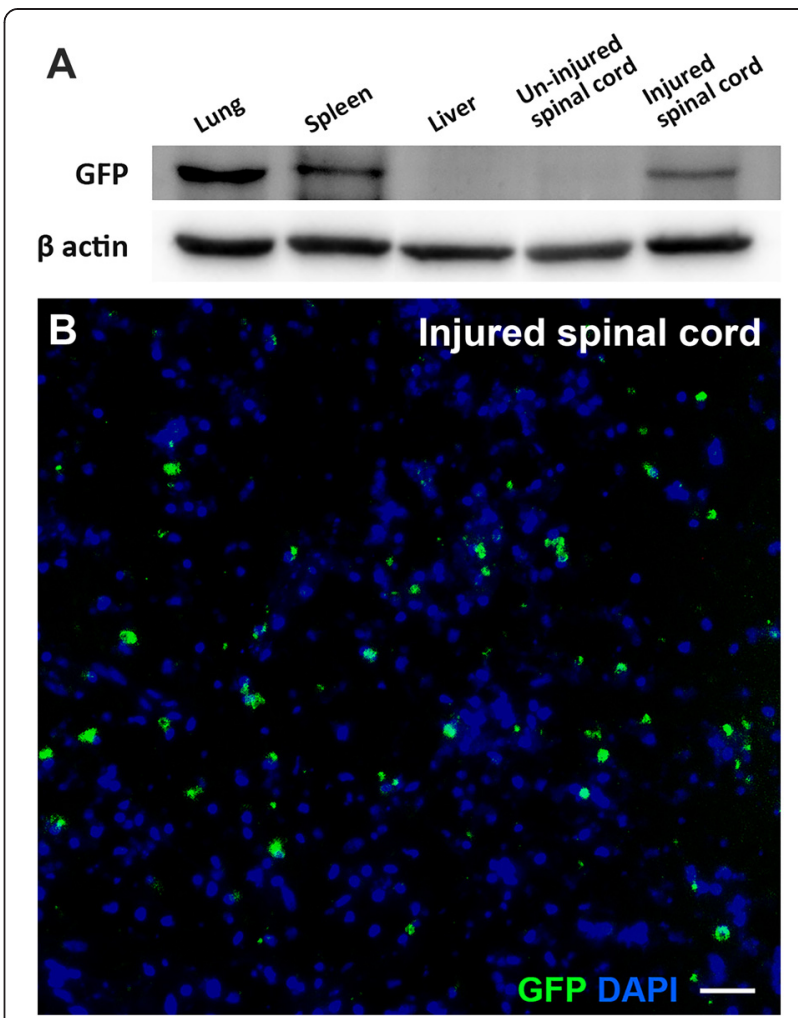

Fig. 2 GFP distribution at 7 days after treatment. a GFP was detected in the lung, spleen, and injured spinal cord at 7 days after intravenous administration of AD-MSCs. However, GFP was not detected in the liver and uninjured normal spinal cord. b GFP-labeled AD-MSCs were observed in the epicenter of the injured spinal cord. Bar: $50 \mu \mathrm{m}$. DAPI 4',6-diamidino-2-phenylindole, GFP green fluorescent protein

\section{Antioxidant effects}

The level of 3-NT (a PN marker) was significantly decreased in the MPSS and AD-MSCs + MPSS groups compared with other groups $(P<0.05$; Fig. 5$)$. The level of 4-HNE (an LP product) was significantly decreased in all treatment groups compared with the control group $(P<0.05)$. In addition, the level of PC (a protein oxidation-related product) was significantly decreased in the AD-MSCs and AD-MSCs + MPSS groups compared with the other two groups $(P<0.05)$.

\section{Anti-inflammatory effects}

A decrease in the level of COX-2 was observed in the MPSS group $(P<0.05)$; however, IL-6 and TNF $\alpha$ levels were not significantly different from that of the control group (Fig. 6a). On the other hand, the levels of COX-2, IL-6, and TNF $\alpha$ were significantly decreased in the ADMSCs and AD-MSCs + MPSS groups when compared with those in the control group $(P<0.05)$.

\section{Astrogliosis and neuronal cells}

The expression of pSTAT3 was significantly decreased in the AD-MSCs and AD-MSCs + MPSS groups when 


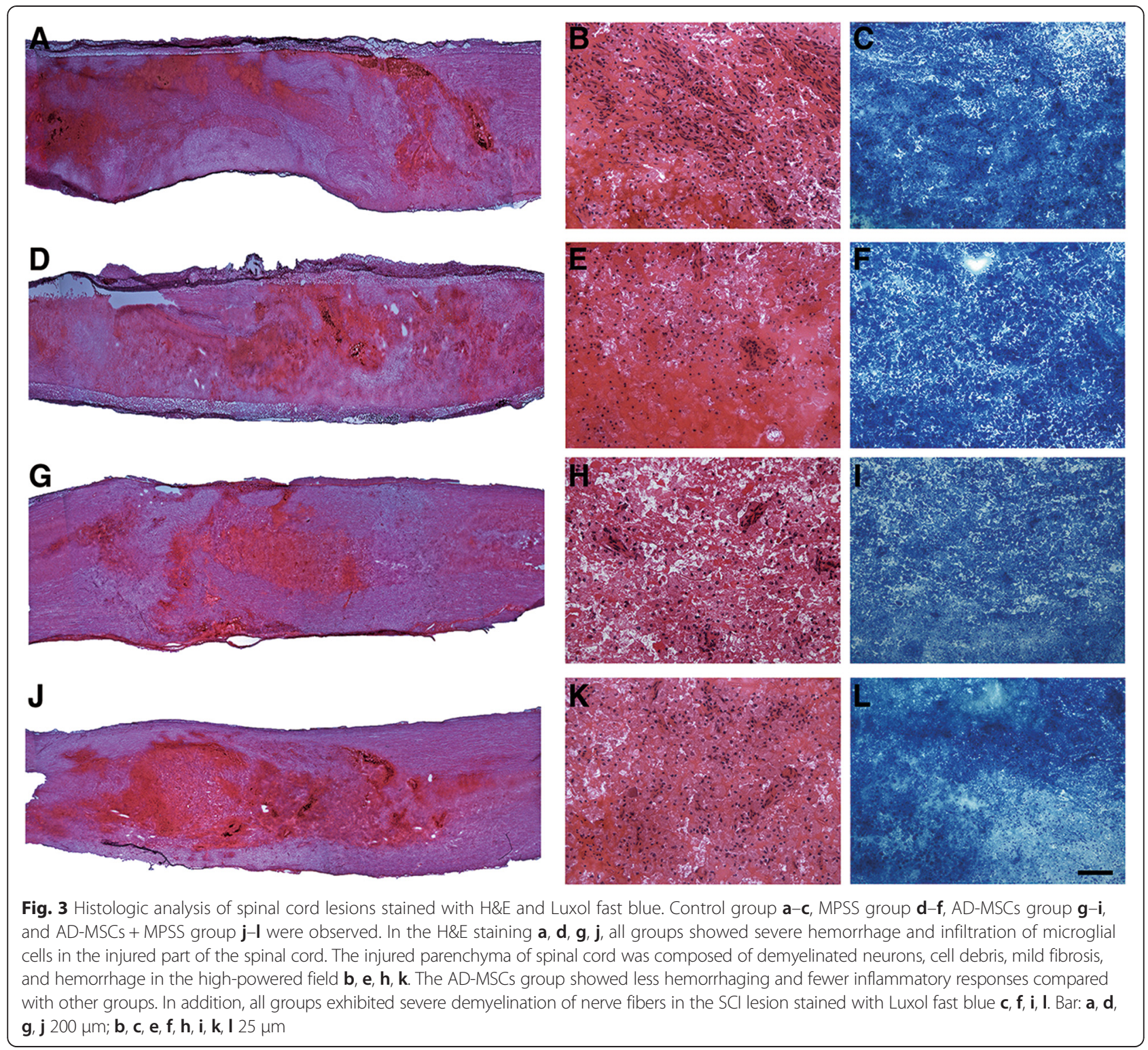

compared with the control group $(P<0.05$; Fig. 6b). In addition, $\beta 3$-tubulin levels were increased in all treatment groups as compared with the control group $(P<0.05)$. The levels of GFAP, a marker of reactive astrocytes, and GalC, a marker of mature oligodendrocytes, were increased in the AD-MSCs and AD-MSCs + MPSS groups when compared with those in the control group.

\section{Discussion}

The objective of this study was to investigate the antioxidant and anti-inflammatory effects of intravenously injected AD-MSCs in an acute SCI model and to compare these effects with those of MPSS, which is used as a neuroprotective agent for the treatment of acute SCI. Administration of AD-MSCs improved hind-limb functions and reduced the occurrence of adverse effects that are associated with high doses of glucocorticoid steroids. MPSS treatment also resulted in some beneficial effects on the clinical signs, but resulted in the adverse effect of gastrointestinal hemorrhage caused by peptic ulceration. This complication may develop because MPSS inhibits phospholipase $\mathrm{A}_{2}$ action, which converts arachidonic acid to COX. These actions of MPSS lead to nonselective inhibition of COX-1 and COX-2. The COX-2 enzyme is involved in inflammatory responses, especially prostaglandin $E_{2}$, but COX-1 is thought to only be involved in normal physiological functions, such as gastrointestinal mucous production, kidney water excretion, and platelet activation [14]. However, AD-MSCs administration did not produce this adverse effect, which suggests that MSCs selectively inhibit COX-2, but not COX-1. It is therefore important to minimize gastrointestinal effects, since they can 

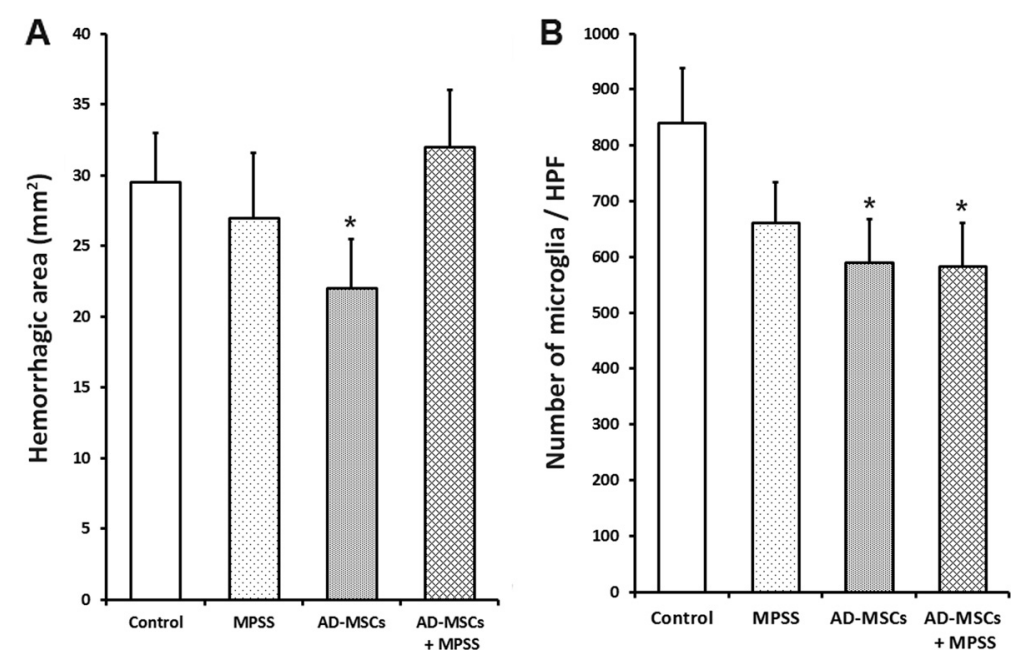

Fig. 4 a The hemorrhagic area was decreased in the AD-MSCs group compared with other groups. $\mathbf{b}$ The infiltration of microglia was decreased in the AD-MSCs and AD-MSCs + MPSS groups. Data presented as mean \pm SD. ${ }^{*} P<0.05$ compared with the control group. AD-MSCs adipose-derived mesenchymal stem cells, HPF high-powered field, MPSS methylprednisolone sodium succinate

result in fatal conditions such as gastrointestinal perforation, anemia, secondary infection, and sepsis if left untreated $[15,16]$.

In the present study, administration of MPSS inhibited nitration and LP, and AD-MSCs inhibited LP and protein oxidation. These antioxidant effects of MPSS correspond well with those reported previously [17]. The effects of MPSS were due to a membrane stabilizing action that inhibits LP by limiting the fluidity of the phospholipids in the neural cell membranes, thereby stunting the LP chain reaction. The MSCs exhibited antioxidant activity through LP prevention, increasing levels of glutathione and superoxide dismutase, and modulating the pathways of antioxidant-related protein activation [18]. Moreover, the histopathological findings in the present study showed that AD-MSCs transplantation reduced intraparenchymal hemorrhage, reduced migration of microglia, and decreased the expression of oxidative metabolites. The reduced hemorrhaging caused by ADMSCs administration might directly reduce the release of free radicals from hematomas in addition to reducing microglial activation. In this study, AD-MSCs and MPSS both showed antioxidant effects, but appeared to do so by different mechanisms. Accordingly, a combination therapy of AD-MSCs and MPSS for acute SCI was required for optimal antioxidation with fewer adverse effects.

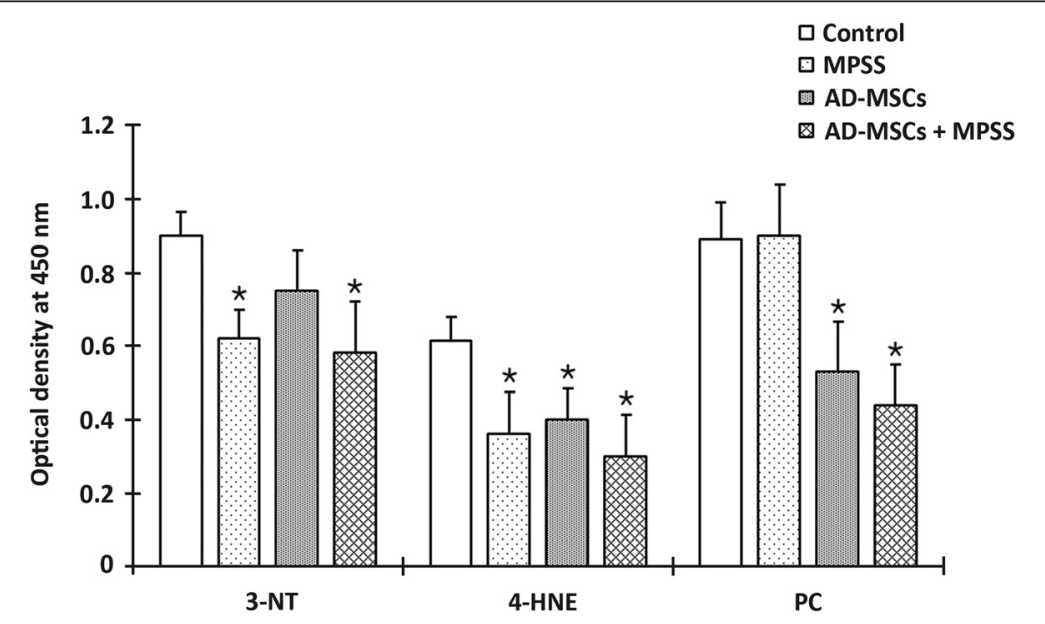

Fig. 5 Oxidant metabolite levels (3-NT, 4-HNE, and PC) at 7 days after treatment. The level of 3-NT was decreased in the MPSS and AD-MSCs + MPSS groups compared with the other groups. The level of 4-HNE was decreased in all treatment groups compared with the control group. In addition, the level of PC was decreased in the AD-MSCs and AD-MSCs + MPSS groups when compared with the other two groups. Data presented as mean \pm SD. ${ }^{P} P<0.05$ compared to the control group. AD-MSCS adipose-derived mesenchymal stem cells, 4-HNE 4-hydroxynenonal, MPSS methylprednisolone sodium succinate, 3-NT 3-nitrotyrosine, $P C$ protein carbonyls 


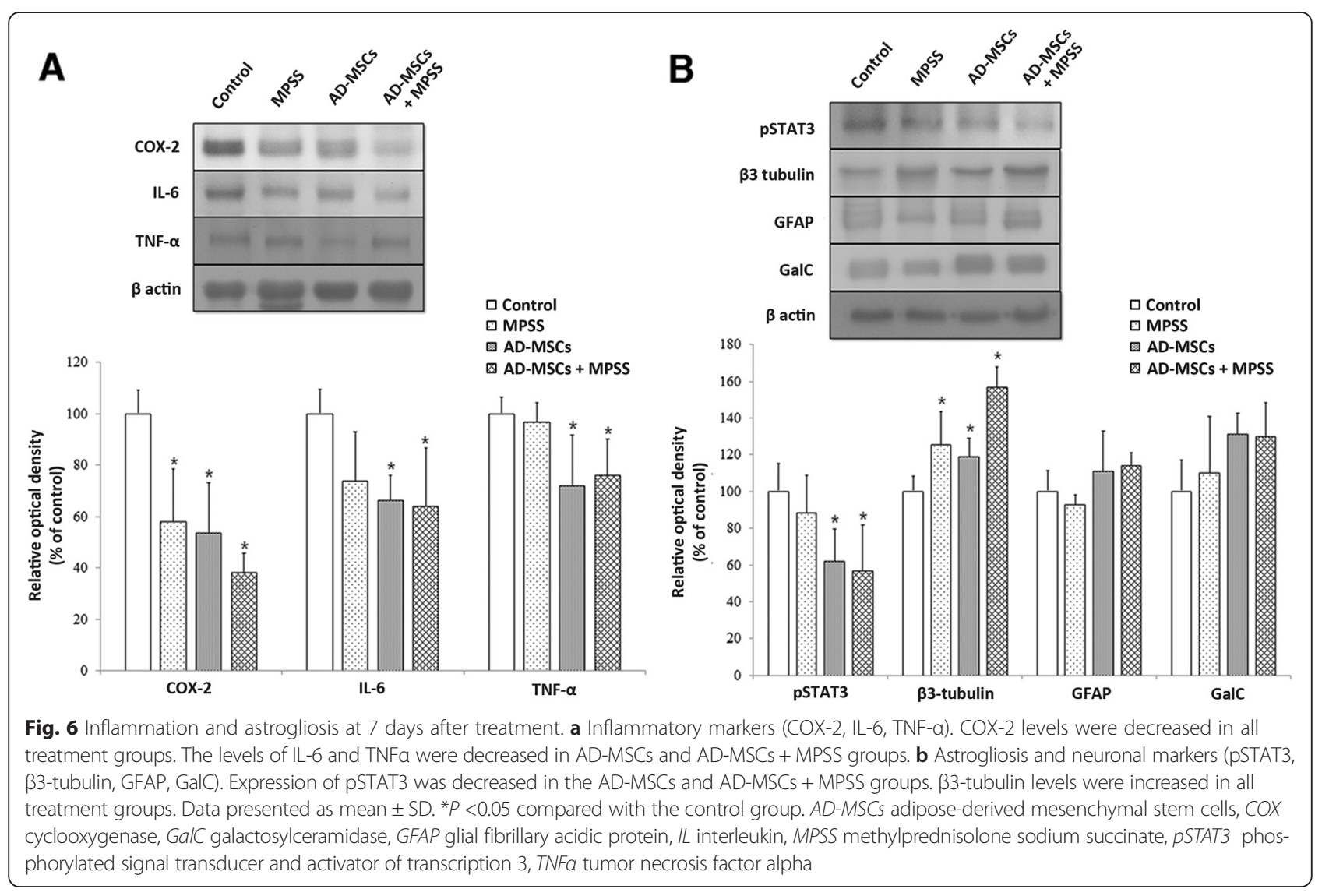

In the present study, intravenous injection of AD-MSCs decreased levels of inflammatory cytokines including COX-2, IL-6, and TNF $\alpha$, which may have resulted from the inhibition of microglial activation and inflammatory responses. However, MPSS does not decrease levels of IL6 and TNF $\alpha$, and it seems that high doses of MPSS exert greater antioxidant effect than anti-inflammatory effects [19]. In addition, the unclear neurological improvement and anti-inflammatory effects of MPSS in the present study might support the results indicating a low therapeutic effect. Previous studies have reported that MSCs exert immunomodulatory effects by attenuating and modulating excessive inflammatory reactions [20, 21]. MSCs have been shown to reduce levels of proinflammatory cytokines such as interferon gamma, TNF $\alpha$, and IL-6 and to increase expression of indoleamine 2,3-dioxygenase, which suppresses T-cell responses and promotes immunological tolerance [22]. In spinal microglia, IL-6 is associated with inflammatory cytokine signaling to induce STAT3, and also has the ability to trigger reactive astrogliosis $[23,24]$. In the present study, AD-MSCs decreased levels of IL-6 which is related to pSTAT3 and astrogliosis, suggesting an anti-astrogliosis effect. Reactive astrocytes contribute to glial scar formation and inhibition of axonal outgrowth. According to recent studies, however, reactive astrocytes also provide beneficial effects that protect adjacent neural tissue and secrete growthpromoting neurotrophic factors [25, 26]. The existing GFAP-expressing reactive astrocytes could create the neuroprotective environment for neurogenesis with transplanted cells.

MSCs transplantation strategies require a safe and efficient method of cellular delivery. In animal models of SCI, the most common delivery method is direct injection into the injured site, which allows many cells to be transplanted effectively, albeit through an extremely invasive procedure that may lead to further injuries. Consequently, this method may be very difficult to implement in human subjects. Therefore, less invasive methods for cell delivery have been investigated and intravenous administration has been identified as an ideal and preferable minimally invasive method for delivering cell transplants for clinical translation. Nonetheless, the actions and cellular distribution of intravenously transplanted cells is a subject of controversy. Previous study showed that transplanted cells even survived for at least several weeks following intravenous transplantation of MSCs in animal models of SCI [27]. However, other studies reported that intravenously transplanted cells were primarily trapped in the lung, and secondarily in the spleen, liver, and kidney, with only a precious few cells found at the injured site [20,28]. In the present study, we detected grafted cells in the lung, spleen, 
and injured spinal cord site, but found no such cells in the uninjured spinal cord after intravenous administration, which shows that the AD-MSCs can probably migrate into the injured spinal cord through the broken blood-spinal cord barrier.

The MSCs therapy was a result of indirect environmental modification rather than direct translineage conversion of migrated MSCs to functional oligodendrocytes or neurons [11]. Transplanted MSCs were able to reduce neurotoxicity and protect cells from apoptosis. MSCs secrete anti-apoptotic protein B-cell lymphoma 2 (Bcl-2), and prevent the release of Bcl-2associated $\mathrm{X}$ protein and caspase 3 , which are proapoptotic proteins $[29,30]$. MSCs also act as neuroprotectors by secreting various angiogenic and neurotrophic factors such as brain-derived neurotrophic factor, nerve growth factor, vascular endothelial growth factor, and hepatocyte growth factor, thereby providing trophic support to damaged neurons [31]. In the present study, intravenous injection of AD-MSCs and/or MPSS increased levels of neuronal markers including $\beta 3$-tubulin and GalC. This could be the result of endogenous neuronal cells that survived in the injured site through the protective effects of AD-MSCs. Furthermore, intravenously injected MSCs show peripheral immunoregulatory properties through inhibition of T-cell activities and through modulation of the host systemic and central nervous system inflammatory responses [32, 33]. It has been suggested that intravenously injected MSCs may not only be involved in systemic immune modulation, but may also migrate to the injured site to exert neuroprotective effects.

\section{Conclusion}

In the current study, it is clear that the spinal cord underwent primary and secondary damages resulting from SCI. In this hostile environment, it is difficult to maintain cell survival, differentiation, and neuronal regeneration. Therefore, it is important to improve the environment by decreasing oxidative damage, LP, and inflammatory responses in order to protect intrinsic neural cells and recover their function. The MPSS treatment in SCI is controversial since it provides only modest neurological benefit despite the risk of serious adverse effects. Our results demonstrated that the intravenous injection of $\mathrm{AD}-\mathrm{MSCs}$ in the acute spinal cord injured dog produced beneficial effects through enhancement of antioxidant and anti-inflammatory activities, and could be used as an alternative treatment modality in acute SCI.

\section{Abbreviations}

AD-MSCs: Adipose-derived mesenchymal stem cells; Bcl-2: B-cell lymphoma 2; COX: Cyclooxygenase; DAPI: 4',6-Diamidino-2-phenylindole; DMEM: Dulbecco's modified Eagle's medium; ELISA: Enzyme-linked immunosorbent assay; FBS: Fetal bovine serum; GalC: Galactosylceramidase; GFAP: Glial fibrillary acidic protein; GFP: Green fluorescent protein; H\&E: Hematoxylin and eosin; 4-HNE: 4-Hydroxynenonal; IL: Interleukin;
LP: Lipid peroxidation; MAC: Minimum alveolar concentration; MPSS: Methylprednisolone sodium succinate; MSC: Mesenchymal stem cell; 3-NT: 3-Nitrotyrosine; PBS: Phosphate-buffered saline; PC: Protein carbonyls; PN: Peroxynitrite; PSTAT3: Phosphorylated signal transducer and activator of transcription 3; SCl: Spinal cord injury; SD: Standard deviation; TBST: 10 mM Tris-HCl, pH 7.6, 150 mM NaCl, 0.05 \% Tween-20; TNFa: Tumor necrosis factor alpha.

\section{Competing interests}

The authors declare that they have no competing interests.

\section{Authors' contributions}

YK and S-HJ were involved in study design and performed experiments, data analysis, and manuscript writing. WHK and O-KK were involved in study design and revised the manuscript. All authors read and approved the manuscript.

\section{Acknowledgements}

This work was partially supported by the Research Institute for Veterinary Science, Seoul National University, and the National Research Foundation of Korea (NRF-2013R1A1A2004506).

Received: 30 April 2015 Revised: 14 August 2015

Accepted: 10 November 2015 Published online: 26 November 2015

\section{References}

1. Oyinbo CA. Secondary injury mechanisms in traumatic spinal cord injury: a nugget of this multiply cascade. Acta Neurobiol Exp (Wars). 2011;71:281-99.

2. Wang J, Rogove AD, Tsirka AE, Tsirka SE. Protective role of tuftsin fragment 1-3 in an animal model of intracerebral hemorrhage. Ann Neurol. 2003;54: 655-64.

3. Wang J, Dore S. Inflammation after intracerebral hemorrhage. J Cereb Blood Flow Metab. 2007;27:894-908.

4. Weiss SJ. Tissue destruction by neutrophils. N Engl J Med. 1989;320:365-76.

5. Bracken MB, Shepard MJ, Collins WF, Holford TR, Young W, Baskin DS, et al. A randomized, controlled trial of methylprednisolone or naloxone in the treatment of acute spinal-cord injury. Results of the Second National Acute Spinal Cord Injury Study. N Engl J Med. 1990;322:1405-11.

6. Bracken MB, Shepard MJ, Holford TR, Leo-Summers L, Aldrich EF, Fazl M, et al. Administration of methylprednisolone for 24 or 48 hours or tirilazad mesylate for 48 hours in the treatment of acute spinal cord injury. Results of the Third National Acute Spinal Cord Injury Randomized Controlled Trial. National Acute Spinal Cord Injury Study. JAMA. 1997;277:1597-604.

7. Hurlbert RJ. Methylprednisolone for the treatment of acute spinal cord injury: point. Neurosurgery. 2014;61 Suppl 1:32-5.

8. Molano Mdel R, Broton JG, Bean JA, Calancie B. Complications associated with the prophylactic use of methylprednisolone during surgical stabilization after spinal cord injury. J Neurosurg. 2002;96:267-72.

9. Park SS, Byeon YE, Ryu HH, Kang BJ, Kim Y, Kim WH, et al. Comparison of canine umbilical cord blood-derived mesenchymal stem cell transplantation times: involvement of astrogliosis, inflammation, intracellular actin cytoskeleton pathways, and neurotrophin-3. Cell Transplant. 2011;20:1867-80.

10. Lim JH, Byeon YE, Ryu HH, Jeong YH, Lee YW, Kim WH, et al. Transplantation of canine umbilical cord blood-derived mesenchymal stem cells in experimentally induced spinal cord injured dogs. J Vet Sci. 2007;8: 275-82.

11. Ryu HH, Lim JH, Byeon YE, Park JR, Seo MS, Lee YW, et al. Functional recovery and neural differentiation after transplantation of allogenic adipose-derived stem cells in a canine model of acute spinal cord injury. J Vet Sci. 2009;10:273-84.

12. Rabinowitz RS, Eck JC, Harper Jr CM, Larson DR, Jimenez MA, Parisi JE, et al. Urgent surgical decompression compared to methylprednisolone for the treatment of acute spinal cord injury: a randomized prospective study in beagle dogs. Spine. 2008;33:2260-8.

13. Bradford MM. A rapid and sensitive method for the quantitation of microgram quantities of protein utilizing the principle of protein-dye binding. Anal Biochem. 1976;72:248-54.

14. Perrone MG, Scilimati A, Simone L, Vitale P. Selective COX-1 inhibition: a therapeutic target to be reconsidered. Curr Med Chem. 2010;17:3769-805.

15. Khan MF, Burks SS, Al-Khayat H, Levi AD. The effect of steroids on the incidence of gastrointestinal hemorrhage after spinal cord injury: a case-controlled study. Spinal Cord. 2014;52:58-60. 
16. Narum S, Westergren T, Klemp M. Corticosteroids and risk of gastrointestinal bleeding: a systematic review and meta-analysis. BMJ Open. 2014;4:e004587. doi: 10.1136/bmjopen-2013-004587.

17. Hall ED. Antioxidant therapies for acute spinal cord injury. Neurotherapeutics. 2011;8:152-67.

18. Lanza C, Morando S, Voci A, Canesi L, Principato MC, Serpero LD, et al. Neuroprotective mesenchymal stem cells are endowed with a potent antioxidant effect in vivo. J Neurochem. 2009:110:1674-84.

19. Hall ED, Springer JE. Neuroprotection and acute spinal cord injury: a reappraisal. NeuroRx. 2004;1:80-100.

20. Seo JH, Jang IK, Kim H, Yang MS, Lee JE, Kim HE, et al. Early immunomodulation by intravenously transplanted mesenchymal stem cells promotes functional recovery in spinal cord injured rats. Cell Med. 2011:2:55-67.

21. Hoogduijn MJ, Popp F, Verbeek R, Masoodi M, Nicolaou A, Baan C, et al. The immunomodulatory properties of mesenchymal stem cells and their use for immunotherapy. Int Immunopharmacol. 2010;10:1496-500.

22. Jui HY, Lin CH, Hsu WT, Liu YR, Hsu RB, Chiang BL, et al. Autologous mesenchymal stem cells prevent transplant arteriosclerosis by enhancing local expression of interleukin-10, interferon-gamma, and indoleamine 2,3dioxygenase. Cell Transplant. 2012;21:971-84.

23. Dominguez E, Rivat C, Pommier B, Mauborgne A, Pohl M. JAK/STAT3 pathway is activated in spinal cord microglia after peripheral nerve injury and contributes to neuropathic pain development in rat. J Neurochem. 2008;107:50-60.

24. Nakamura M, Okada S, Toyama Y, Okano H. Role of IL-6 in spinal cord injury in a mouse model. Clin Rev Allergy Immunol. 2005;28:197-204.

25. Lukovic D, Stojkovic M, Moreno-Manzano V, Jendelova P, Sykova E, Bhattacharya SS, et al. Concise review: reactive astrocytes and stem cells in spinal cord injury: good guys or bad guys? Stem Cells. 2015;33:1036-41.

26. Burda JE, Bernstein AM, Sofroniew MV. Astrocyte roles in traumatic brain injury. Exp Neurol. 2015; doi: 10.1016/j.expneurol.2015.03.020.

27. Sykova $E$, Jendelova P. Migration, fate and in vivo imaging of adult stem cells in the CNS. Cell Death Differ. 2007:14:1336-42.

28. Takahashi Y, Tsuji O, Kumagai G, Hara CM, Okano HJ, Miyawaki A, et al. Comparative study of methods for administering neural stem/progenitor cells to treat spinal cord injury in mice. Cell Transplant. 2011;20:727-39.

29. Okazaki T, Magaki T, Takeda M, Kajiwara Y, Hanaya R, Sugiyama K, et al. Intravenous administration of bone marrow stromal cells increases survivin and $\mathrm{BCl}-2$ protein expression and improves sensorimotor function following ischemia in rats. Neurosci Lett. 2008;430:109-14.

30. Wang SP, Wang ZH, Peng DY, Li SM, Wang H, Wang XH. Therapeutic effect of mesenchymal stem cells in rats with intracerebral hemorrhage: reduced apoptosis and enhanced neuroprotection. Mol Med Rep. 2012;6:848-54.

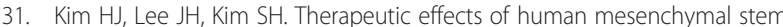
cells on traumatic brain injury in rats: secretion of neurotrophic factors and inhibition of apoptosis. J Neurotrauma. 2010;27:131-8.

32. Ben-Hur T. Immunomodulation by neural stem cells. J Neurol Sci. 2008;265: 102-4.

33. Zappia E, Casazza S, Pedemonte E, Benvenuto F, Bonanni I, Gerdoni E, et al. Mesenchymal stem cells ameliorate experimental autoimmune encephalomyelitis inducing T-cell anergy. Blood. 2005;106:1755-61.

\section{Submit your next manuscript to BioMed Central and take full advantage of:}

- Convenient online submission

- Thorough peer review

- No space constraints or color figure charges

- Immediate publication on acceptance

- Inclusion in PubMed, CAS, Scopus and Google Scholar

- Research which is freely available for redistribution

Submit your manuscript at www.biomedcentral.com/submit 